\title{
Software Defined Radio for Smart Utility Networks
}

\author{
Srinivas Lingam
}

\author{
Timothy Schmidl \\ Texas Instruments \\ 13532 North Central Expressway \\ Dallas, TX, USA \\ \{lsri, schmidl, batra\}@ti.com
}

\begin{abstract}
In this paper, we describe a software defined radio (SDR) platform that can be used to implement the IEEE 802.15.4g standard for low data rate wireless smart utility networks (SUN). The SUN standard supports multiple wireless band plans, defines multiple physical (PHY) layers: FSK, OFDM and O-QPSK, each supporting a large number of data rates. Because of the flexibility allowed in the standards, an SDR approach provides the greatest flexibility in implementing the standard. We propose to use the SDR platform based on Texas Instruments C28x 32-bit MCU platform and a sub-1GHz CC1260 low power integrated radio. This platform may be used for both the smart e-meter as well as the data concentrator. We begin the paper by providing an overview of the different PHY layers and enumerating the common elements across all PHYs for both the transmitter and receiver. Next, we discuss a partitioning between software and hardware that provides the flexibility without sacrificing efficiency. Finally, we provide the details of a hardware accelerator incorporated into the C28x architecture (VCU II) that can simplify many of the complex tasks associated with these PHYs.
\end{abstract}

\section{Categories and Subject Descriptors}

C.2.1 [Computer-Communication Networks]: Network Architecture and Design - Wireless Communication. C.3 [Special-Purpose and Application-based Systems]: Signal Processing Systems.

\section{General Terms}

Algorithms, Measurement, Performance, Design.

Keywords

SDR; Software Radio; Software Defined Radio; Wireless; OFDM; FSK; O-QPSK; DSSS; IEEE 802.15.4g; SUN; Smart Grid; Smart Utility Networks.

\section{INTRODUCTION}

The IEEE 802.15.4g standard describes a physical layer for lowpower, low-data rate smart utility networks [1]. This standard was developed by technical experts representing utilities, smart meter manufacturers and semiconductor companies. The final standard defines multiple wireless band plans, multiple PHY layers, each supporting a large number of possible data rates. The flexibility in the standard enables backwards compatibility with existing FSK

Permission to make digital or hard copies of all or part of this work for personal or classroom use is granted without fee provided that copies are not made or distributed for profit or commercial advantage and that copies bear this notice and the full citation on the first page. Copyrights for components of this work owned by others than ACM must be honored. Abstracting with credit is permitted. To copy otherwise, or republish, to post on servers or to redistribute to lists, requires prior specific permission and/or a fee. Request permissions from permissions@acm.org.

SRIF'14, August 18, 2014, Chicago, Illinois, USA

Copyright (C) 2014 ACM 978-1-4503-2995-8/14/08...\$15.00.

http://dx.doi.org/10.1145/2627788.2627797 proprietary solutions that had been developed and deployed by metering manufactures and utilities.

In addition to focusing on backwards compatibility, this standard also defines two additional PHYs (OFDM and O-QPSK) that focus on supporting higher data rates and increasing spectral efficiency when compared to existing proprietary solutions. Over time, the increased data rate will be needed to support increased data traffic as well as complex two-way communications needed to support variable pricing. From an implementation perspective, only the FSK PHY with a symbol of $50 \mathrm{ksps}$ is mandatory. The remaining FSK symbol rates as well as the other PHYs (OFDM and O-QPSK) are optional.

This IEEE 802.15.4g standard supports a number of frequency bands, from $169 \mathrm{MHz}$ to $2.4 \mathrm{GHz}$, and channel bandwidths, from 12.5 - $600 \mathrm{kHz}$ for FSK, $200-1200 \mathrm{kHz}$ for OFDM, and $200-$ $2000 \mathrm{kHz}$ for O-QPSK, to enable deployment of smart utility networks in different regions across the world. A large number of data rates, from 2.4 - $400 \mathrm{kbps}$ for FSK, 50 - $800 \mathrm{kbps}$ for OFDM and 6.25 - $500 \mathrm{kbps}$ for O-QPSK, are also supported by this standard. This large range of data rates enables multiple applications, including those that require low data rates and long range as well as those that require high data rates and short range [1]. In fact, the flexibility in the standard allows support for inhome applications that require distances of just a few feet to those rural applications that require distances of a few miles [2].

This paper is organized as follows. Section 2 demonstrates the need for a software-defined radio platform to address the SUN market. Section 3 provides an overview of the different PHY layers in the IEEE 802.15.4g standard. Section 4 shows the details of the SDR architecture, while Section 5 enumerates the complexity of several key algorithms used in the PHY layer. Finally, the conclusions are summarized in Section 6.

\section{SDR FOR SUN}

A smart e-meter has a typical lifespan of 15 to 20 years before it is replaced by the utility. A software-defined radio provides the flexibility to support multiple physical layers, such as those defined in IEEE 802.15.4g, as well as any evolutions to the standard. Enhancements to physical layers or potentially new, unforeseen, standards can be pushed to the e-meter via over-theair updates. Updating the software over-the-air is an attractive proposition because it eliminates the need of a truck roll to update the meter manually.

Another advantage of the SDR platform is that the software can be designed to support proprietary solutions that have already been deployed in the legacy AMI networks. Since most utilities have already developed legacy networks, having the flexibility to communicate with meters in these networks can save the utility company significant amount of money. 
High-end SDR solutions targeting LTE and WLAN solutions that support data rates in excess of $100 \mathrm{Mbps}$ typically require heterogeneous multi-core solutions based on a combination of multiple cores consisting of DSPs, application-specific instruction processors (ASIPs) and MCUs with potentially sophisticated mapping tools [3]. Since the SUN standard supports data rates below $1 \mathrm{Mbps}$, it can be implemented using a single core DSP with an ASIP that accelerates very specific instructions [4]. This type of programmable approach has been used successfully in Narrowband OFDM Power Line Communications (PLC) to address the multiple standards and fragmented market [5].

\section{MULTIPLE PHY LAYER OVERVIEW}

In this section, the three physical layers defined in IEEE 802.15.4g are described. There are multiple frequency bands available for e-metering applications throughout the world, so each physical layer was defined to have the flexibility to be deployed in many of these bands. The term "multi-rate and multiregional" is abbreviated as MR, so the three PHYs are denoted as MR-FSK, MR-OFDM, and MR-O-QPSK (which is also known as direct-sequence spread-spectrum or DSSS).

\subsection{MR-FSK}

Each MR-FSK packet consists of a synchronization header (SHR) that contains a preamble and a start frame delimiter (SFD) followed by a PHY header (PHR) and a PHY payload or PSDU. The preamble is a repetitive "01010101" pattern for 2-FSK or "0111011101110111" for 4-FSK. The SFD is one of two 16-bit patterns for 2-FSK and is used to indicate whether a $K=4$, rate $1 / 2$ convolution code is used. For 4-FSK, the SFD is one of two 32bit patterns.

The PHR contains 16 information bits, which includes 1 bit to indicate a mode switch, 1 bit to indicate the length of the frame check sequence (2 or 4 bytes), 1 bit to indicate whether data whitening (with a 511 length PN9 sequence) is used, 11 bits to indicate the frame length in octets (max payload size is 2047 octets) and 2 reserved bits.

The convolutional code can be implemented either as a nonrecursive, non-systematic code or alternatively as a recursive, systematic code. The code protects both concatenation of the PHR and the PSDU. For the $902-928 \mathrm{MHz}$ ISM band in the United States, the mandatory MR-FSK mode is $50 \mathrm{ksps}$ 2-FSK with modulation index 1 and channel spacing of $200 \mathrm{kHz}$ and the two named optional modes are 150 ksps 2-FSK and 200 ksps 2FSK, both of which have modulation index 0.5 and channel spacing of $400 \mathrm{kHz}$. When the convolutional code is used, a $4 \times 4$ interleaver operating on pairs of output bits from the encoder is also used.

A generic PHY is also defined for MR-FSK that allows a large degree of flexibility as shown in Figure 1. The modulation order can be either 2-FSK or 4-FSK, the modulation index can vary from 0.25 to 2.5 in increments of 0.05 , the BT value can be either 0.5 or 1.0 , and the symbol rate can be any integer from 1 to $2,000,000$. There are literally hundreds of millions of combinations that can be implemented, so it would be impractical to build and test a device that supports all possible combinations. However in practice, the number of combinations that are expected to be deployed will likely be a single digit.

\begin{tabular}{|c|c|c|c|c|c|c|c|c|}
\hline \multicolumn{3}{|c|}{ Octets: 1} & \multicolumn{2}{|c|}{1} & 4 & 2 & 4 & 4 \\
\hline Bit it: $0-1$ & $2-3$ & $4-7$ & Bit it: $0-5$ & $6-7$ & \multirow[b]{2}{*}{ 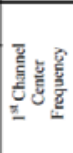 } & \multirow[b]{2}{*}{ 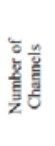 } & \multirow[b]{2}{*}{ 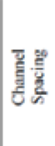 } & \multirow[b]{2}{*}{ 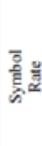 } \\
\hline 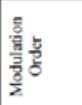 & 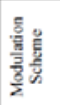 & 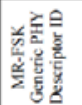 & 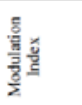 & $\bar{m}$ & & & & \\
\hline
\end{tabular}

Figure 1. Generic PHY Descriptor Format [1]

There is also an optional mode switch packet that begins with MR-FSK and then changes to a different modulation for the remainder of the packet as shown in Figure 2. This is one way to ensure that multiple PHY modes can coexist in the same network. In this case, the signaling to switch between modulations is implemented at the physical layer.

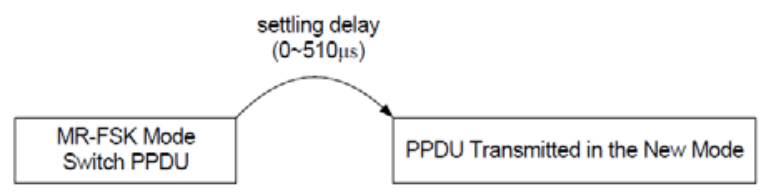

Initiate Mode Switch

PPDU with payload that is transmitted in new mode Figure 2. Mode Switch Format [1]

\subsection{MR-OFDM}

The MR-OFDM PHY is optional to implement, but if it is implemented, then the BPSK and QPSK modulation schemes are mandatory with 16-QAM being optional. Each OFDM symbol includes a cyclic prefix of duration $24 \mu$ s followed by a base symbol of duration $96 \mu$ s for a total symbol duration of $120 \mu \mathrm{s}$. The subcarrier spacing is $31250 / 3 \mathrm{~Hz}$, and the symbol rate is $25 / 3$ ksps. The supported data rates range from $50 \mathrm{kbps}$ to $800 \mathrm{kbps}$, with a spectral efficiency that is much higher than either MR-FSK or MR-O-QPSK.

There are four OFDM options defined in the standard as shown in Figure 3. These four options differ primarily in the DFT size or the total number of tones. When the DFT size decreases by a factor of two, the number of active tones also decreases by approximately a factor of two.

\begin{tabular}{|l|c|c|c|c|}
\hline \multicolumn{1}{|c|}{ Parameter } & $\begin{array}{c}\text { OFDM } \\
\text { Option 1 }\end{array}$ & $\begin{array}{c}\text { OFDM } \\
\text { Option 2 }\end{array}$ & $\begin{array}{c}\text { OFDM } \\
\text { Option 3 }\end{array}$ & $\begin{array}{c}\text { OFDM } \\
\text { Option 4 }\end{array}$ \\
\hline Nominal bandwidth (kHz) & 1094 & 552 & 281 & 156 \\
\hline Channel spacing (kHz) & 1200 & 800 & 400 & 200 \\
\hline DFT size & 128 & 64 & 32 & 16 \\
\hline Active tones & 104 & 52 & 26 & 14 \\
\hline \# Pilot tones & 8 & 4 & 2 & 2 \\
\hline \# Data tones & 96 & 48 & 24 & 12 \\
\hline $\begin{array}{l}\text { MCS0 (kb/s) } \\
\text { (BPSK rate 1/2 with 4x frequency repetition) }\end{array}$ & 100 & 50 & - & - \\
\hline $\begin{array}{l}\text { MCS1 (kb/s) } \\
\text { (BPSK rate 1/2 with 2x frequency repetition) }\end{array}$ & 200 & 100 & 50 & - \\
\hline $\begin{array}{l}\text { MCS2 (kb/s) } \\
\text { (QPSK rate 1/2 and 2x frequency repetition) }\end{array}$ & 400 & 200 & 100 & 50 \\
\hline $\begin{array}{l}\text { MCS3 (kb/s) } \\
\text { (QPSK rate 1/2) }\end{array}$ & 800 & 400 & 200 & 100 \\
\hline $\begin{array}{l}\text { MCS4 (kb/s) } \\
\text { (QPSK rate 3/4) }\end{array}$ & - & 600 & 300 & 150 \\
\hline $\begin{array}{l}\text { MCS5 (kb/s) } \\
\text { (16-QAM rate 1/2) }\end{array}$ & - & 800 & 400 & 200 \\
\hline $\begin{array}{l}\text { MCS6 (kb/s) } \\
\text { (16-QAM rate 3/4) }\end{array}$ & - & - & 600 & 300 \\
\hline
\end{tabular}

Figure 3. Numerology for MR-OFDM Options 1-4 [1]. 
The MR-OFDM packet also consists of a SHR, PHR, and PSDU with the main difference being in the SHR, which contains multiple short training field (STF) symbols followed by two long training field (LTF) symbols. The structure of multiple STF symbol is shown in Figure 4. There are four STF symbols, and each symbol is formed by repeating a short sequence in the time domain. The last half of the last base symbol of the STF is negated in the time-domain. This negation can aid the receiver in detection of the transition between the STF symbols and the LTF symbols.

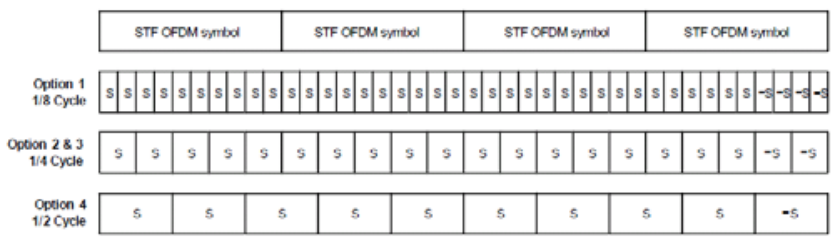

Figure 4. Structure of STF Symbols for MR-OFDM [1]

There are two LTF symbols that follow the four STF symbols. A double length cyclic prefix precedes two base symbols. This creates a continuous waveform throughout both LTF symbols that can aid the receiver in synchronizing to the received MR-OFDM packet and estimating the channel response.
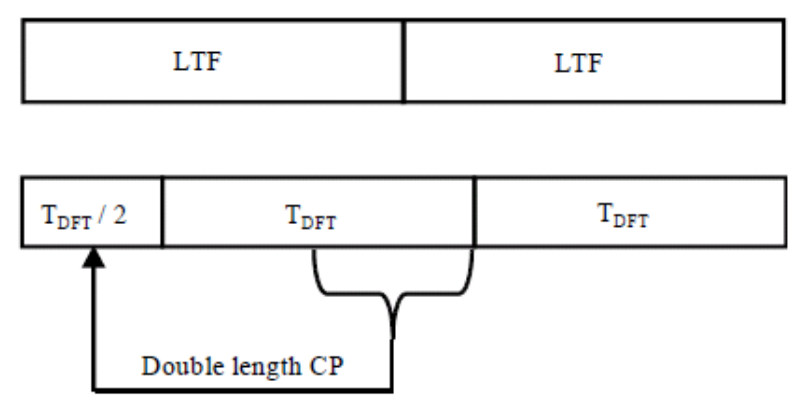

Figure 5. Structure of LTF Symbols for MR-OFDM [1]

Figure 6 shows the structure of the PHR. The rate field indicates the modulation and coding scheme (MCS) that is being used for the current packet. The frame length field indicates the number of octets in the PSDU with a maximum size of 2047 octets. The PHR also indicates which of the four possible scrambler seeds is being used for the payload and includes an 8-bit header check sequence (HCS) that can be used to detect for errors in the received header. Typically, the receiver discards the packet if the HCS fails. There are six tail bits that are used to terminate the $\mathrm{K}=7$ convolutional code. The convolutional code is then reset for the PSDU.

\begin{tabular}{|c|c|c|c|c|c|c|c|c|}
\hline $\begin{array}{c}\text { Bit string } \\
\text { index }\end{array}$ & $0-4$ & 5 & $6-16$ & $17-18$ & $19-20$ & 21 & $22-29$ & $30-35$ \\
\hline $\begin{array}{c}\text { Bit } \\
\text { mapping }\end{array}$ & $\mathrm{RA}_{4}-\mathrm{RA}_{0}$ & $\mathrm{R}$ & $\mathrm{L}_{10}-\mathrm{L}_{0}$ & $\mathrm{R}_{1}-\mathrm{R}_{0}$ & $\mathrm{~S}_{1}-\mathrm{S}_{0}$ & $\mathrm{R}$ & $\mathrm{H}_{7}-\mathrm{H}_{0}$ & $\mathrm{~T}_{5}-\mathrm{T}_{0}$ \\
\hline $\begin{array}{c}\text { Field } \\
\text { name }\end{array}$ & Rate & Reserved & $\begin{array}{c}\text { Frame } \\
\text { Length }\end{array}$ & Reserved & Scrambler & Reserved & HCS & Tail \\
\hline
\end{tabular}

Figure 6. Structure of PHR for MR-OFDM [1]

The rate $1 / 2$ convolutional encoder is shown in Figure 7. This code is much stronger than the one used for MR-FSK.

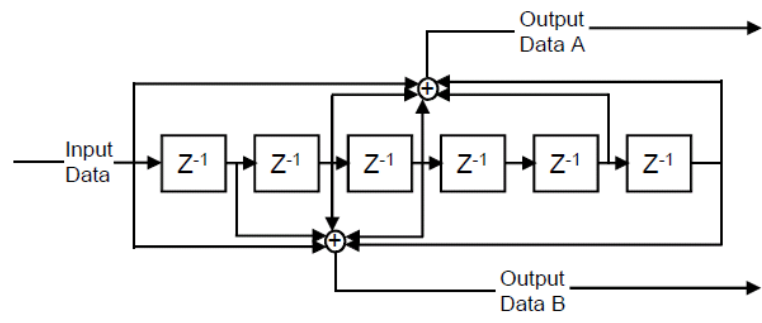

Convolutional Encoder: Rate 1/2, constraint length $\mathrm{K}=7$ Octal generator polynomials $[133,171]$

Figure 7. Convolutional Encoder for MR-OFDM [1]

The minimum performance requirements for sensitivity are shown in Figure 8. Note that there are approximately $3 \mathrm{~dB}$ steps in the required signal power levels between MCS levels for an AWGN channel.

\begin{tabular}{|l|c|c|c|c|}
\hline & Option 1 & Option 2 & Option 3 & Option 4 \\
\hline MCS0 (BPSK 1/2 rate coded and 4x frequency repetition) & $-103 \mathrm{dBm}$ & $-105 \mathrm{dBm}$ & - & - \\
\hline MCS1 (BPSK 1/2 rate coded and 2x frequency repetition) & $-100 \mathrm{dBm}$ & $-103 \mathrm{dBm}$ & $-105 \mathrm{dBm}$ & - \\
\hline MCS2 (QPSK 1/2 rate coded and 2x frequency repetition) & $-97 \mathrm{dBm}$ & $-100 \mathrm{dBm}$ & $-103 \mathrm{dBm}$ & $-105 \mathrm{dBm}$ \\
\hline MCS3 (QPSK $1 / 2$ rate coded) & $-94 \mathrm{dBm}$ & $-97 \mathrm{dBm}$ & $-100 \mathrm{dBm}$ & $-103 \mathrm{dBm}$ \\
\hline MCS4 (QPSK $3 / 4$ rate coded) & - & $-94 \mathrm{dBm}$ & $-97 \mathrm{dBm}$ & $-100 \mathrm{dBm}$ \\
\hline MCS5 (16-QAM 1/2 rate coded) & - & $-91 \mathrm{dBm}$ & $-9.4 \mathrm{dBm}$ & $-97 \mathrm{dBm}$ \\
\hline MCS6 (16-QAM $/ 4$ rate coded) & - & - & $-91 \mathrm{dBm}$ & $-94 \mathrm{dBm}$ \\
\hline
\end{tabular}

Figure 8. Min. Sensitivity Requirements for MR-OFDM [1].

\subsection{MR-O-QPSK}

The MR-O-QPSK or DSSS PHY is also optional to implement. As with the other PHYs, each packet consists of an SHR, PHR, and PSDU. The SHR consists of a preamble and SFD. Figure 9 shows the chip rate and spreading factor for the SHR as a function of the frequency band. For sub- $1 \mathrm{GHz}$ operation, the chip rates are limited to 100 and $1000 \mathrm{kchip} / \mathrm{s}$.

\begin{tabular}{|c|c|c|c|}
\hline $\begin{array}{c}\text { Frequency band } \\
\text { (MHz) }\end{array}$ & $\begin{array}{c}\text { Chip rate } \\
\text { (kchip/s) }\end{array}$ & BDE & Spreading \\
\hline $470-510$ & 100 & yes & $(32,1)_{0}$-DSSS \\
\hline $779-787$ & 1000 & yes & $(64,1)$-DSSS \\
\hline $868-870$ & 100 & yes & $(32,1)_{0}$-DSSS \\
\hline $902-928$ & 1000 & yes & $(64,1)$-DSSS \\
\hline $917-923.5$ & 1000 & yes & $(64,1)$-DSSS \\
\hline $920-928$ & 100 & yes & $(32,1)_{0 / 1}$-DSSS \\
\hline $950-958$ & 100 & yes & $(32,1)_{0}$-DSSS \\
\hline $2400-2483.5$ & 2000 & yes & $(128,1)$-DSSS \\
\hline
\end{tabular}

Figure 9. SHR Coding, Spreading Parameters for MR-OQPSK [1]

Each baseband chip is represented by a half-sine pulse shape. For a bit on the in-phase (I) branch, the pulse duration is two chip periods. For a bit on the quadrature $(\mathrm{Q})$ branch, the duration is also two chip periods but with an offset of one chip period in time. Hence the modulation is termed offset QPSK (O-QPSK). A $\mathrm{K}=7$, rate $1 / 2$ convolutional code is used for all data rates. Figure 10 shows the rate modes defined for the $902-928 \mathrm{MHz}$ band. The lower rate modes have spreading factors of 16,4 , and 2 , while the highest rate mode (RM3) does not use spreading but supports a data rate of $500 \mathrm{kbps}$. Rate Mode 0 is the only rate mode that uses bit differential encoding (BDE). 


\begin{tabular}{|c|c|c|c|c|c|c|}
\hline $\begin{array}{l}\text { Frequency } \\
\text { band }(\mathrm{MHz})\end{array}$ & $\begin{array}{l}\text { Chip rate } \\
\text { (kchip/s) }\end{array}$ & Rate:Node & BDE & Spreadling & $\begin{array}{c}\text { rate }^{1 / 2} \\
\text { FEC + } \\
\text { interleaver }\end{array}$ & $\begin{array}{c}\text { Data rate } \\
(\mathbf{k b} / \mathrm{s})\end{array}$ \\
\hline \multirow[t]{4}{*}{$902-928$} & \multirow[t]{4}{*}{1000} & 0 & yes & $(16,1)_{01}$-DSSS & yes & 31.25 \\
\hline & & 1 & no & $(16,4)$-DSSS & yes & 125 \\
\hline & & 2 & no & (8.4)-DSSS & yes & 250 \\
\hline & & 3 & no & none & yes & 500 \\
\hline
\end{tabular}

Figure 10. Rate Modes for MR-O-QPSK [1]

Figure 11 shows the structure of the PHR, where the spreading mode bit is set to 0 to indicate that DSSS (direct sequence spread spectrum) is used, two bits are used to indicate the rate mode of the packet, and eleven bits are used to indicate the PSDU length in octets, up to 2047 octets, and an 8-bit HCS is used to detect for errors in the header.

\begin{tabular}{|c|c|c|c|c|c|c|c|}
\hline Bit string index & 0 & 1 & 2 & 3 & 4 & $5-15$ & $16-23$ \\
\hline Bit mapping & $\mathrm{SM}$ & $\mathrm{RM}_{1}$ & $\mathrm{RM}_{0}$ & $\mathrm{R}_{1}$ & $\mathrm{R}_{0}$ & $\mathrm{~L}_{10}-\mathrm{L}_{0}$ & $\mathrm{H}_{7}-\mathrm{H}_{0}$ \\
\hline Field name & Spreading Mode & Rate Mode & Reserved & Frame Length & HCS \\
\hline
\end{tabular}

Figure 11. PHR for MR-O-QPSK [1]

\section{SDR ARCHITECTURE}

Figure 12 shows the overall architecture of the SDR platform, which consists of a fully integrated quadrature RF front-end with digitized baseband signal input and output and a DSP whose computational efficiency is enhanced though an ASIP. The RF front-end operates in the sub- $1 \mathrm{GHz}$ band and is a member of Texas Instruments' Performance Line RF transceiver family. The $\mathrm{DSP} / \mathrm{MCU}$ is a dual-core processor that is a member of the TMS320F28x family [6]. Separate interfaces are used for control and data.

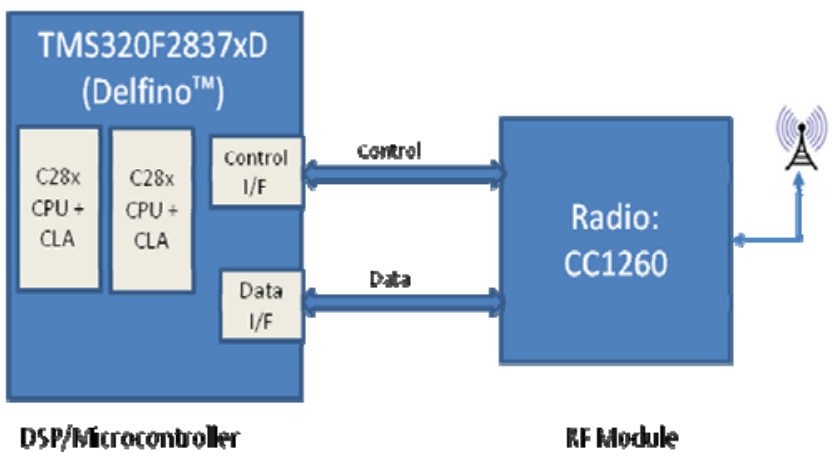

Figure 12. SDR Block Diagram for IEEE 802.15.4g

The radio provides support for multiple frequency bands, channel bandwidths and TX and RX sampling rates as defined in the IEEE 802.15.4g standard, while the DSP/MCU provides the ability to implement flexible signal processing algorithms. Channel filtering is achieved through the combination of the integrated filters on the quadrature RF front-end and filtering in the DSP.

\subsection{Overview of Radio (CC1260)}

The CC1260 radio is a high performance and low power quadrature RF front-end with a digitized baseband signal interface targeting SDR systems that supports sub-1 GHz frequency bands. This radio is well suited for applications, such as smart metering (AMR/AMI), home and building automation, wireless alarm and security systems, and IEEE 802.15.4g applications. The radio was designed to be cost effective, while still delivering excellent performance at very low power and low voltage operation.

\subsubsection{Radio to DSP/MCU Interface}

The CC1260 supports two interfaces with the DSP/MCU. The 4wire SPI interface is used to configure registers and read status information. The bi-directional data interface allows a continuous stream of data in bytes to be transferred between the DSP/MCU and radio. The data interface supports multiple samplings in both directions.

\subsubsection{Radio Transmit Configuration}

The CC1260 has a high performance linear RF front-end supporting programmable output power up to $+14 \mathrm{dBm}$ with a very fine step size, which is accomplished by adjusting the digital I/Q gain, DAC, TX mixer, and power amplifier backoffs. The radio also has a programmable TX mixer, TX filter bandwidth, and input sampling rate for the TX DAC. Additionally, the radio supports a smooth power ramp that improves spectral efficiency of the system by reducing the out-of-band signal energy created by abrupt changes in the output power. Smooth power ramp is applied to the I/Q samples at the start and end of a transmission.

The radio also supports TX calibration via I/Q gain and phase correction. In addition, the calibration can be improved through an optional mechanism that rounds the DAC output samples and shapes the noise produced by the quantization error.

\subsubsection{Radio Receive Configuration}

The CC1260 receive chain supports a programmable anti-aliasing filter that is implemented by concatenating a programmable decimation CIC filter with a programmable FIR filter. The receive chain also supports an ADC whose sampling rate is programmable, DC offset removal, quadrature imbalance correction, and receiver gain control. The gain control provides a gain range of $85 \mathrm{~dB}$.

\subsection{Processor (C28x)}

The DSP/MCU used in the SDR platform is a part of Texas Instruments' F2837xD Delfino ${ }^{\mathrm{TM}}$ family. This dual-core DSP/MCU has integrated analog features, communications interfaces and is based on Texas Instruments' 32-bit C28x floating-point CPU core. Each of the C28x cores has a maximum clock rate of $200 \mathrm{MHz}$, which is sufficient for most of the SUN applications.

The compute capability of the C28x cores is enhanced with additional accelerators like trigonometric math unit (TMU) and Viterbi complex unit (VCU II). Both of these application-specific instruction set processor (ASIP) accelerators are specifically targeted for accelerating $\mathrm{PHY}$ algorithms. 


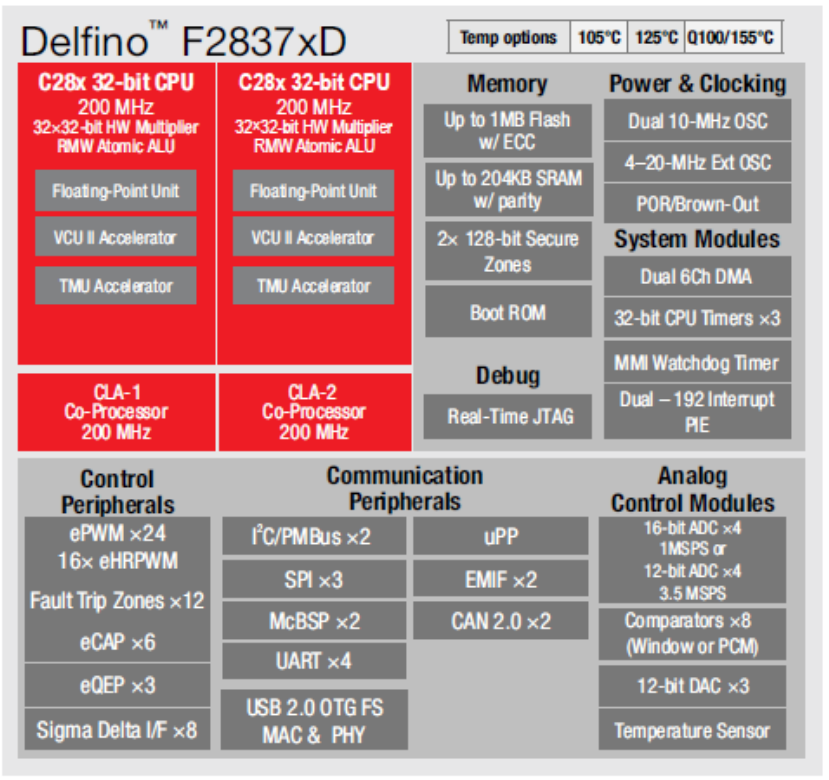

Figure 13. DSP (C28x) Functional Block Diagram

Each of the C28x cores also includes an independent floatingpoint control law accelerator (CLA), which can be used to manage independent control loops, signal pre-processing, and DSP filtering on both the TX and RX chains of the PHY modem. Along with the CLA, the device can provide 800 MIPS of total system performance [6].

The device also supports security enhanced on-chip flash of up to $1024 \mathrm{~KB}$, which can be used to store multiple modems on the same device. The architecture of this DSP is illustrated in Figure 13. Note that this device includes multiple channels of direct memory access (DMA) to allow for data movements to/from the radio while CLA and C28x are processing prior blocks of data.

\subsubsection{Viterbi Complex Math Unit (VCU II)}

The VCU II is an enhancement of the VCU that was initially introduced in the F28M35x Concerto ${ }^{\mathrm{TM}}$ family of devices. The VCU is tightly integrated into the C28x CPU's pipeline. The application issues instructions to the VCU using dedicated registers with pointers to data and result buffers. This allows the application to utilize VCU-accelerated instructions at any time.

The VCU can significantly improve the performance of several communications algorithms, up to a factor of $7 \times$ [4][5], thereby reducing the MIPS requirement on the DSP/MCU. It also enables devices to operate either at a lower clock speed, thereby reducing power consumption, or implement additional processing or more complicated algorithms.

\section{VCU enhances complex math capabilities}

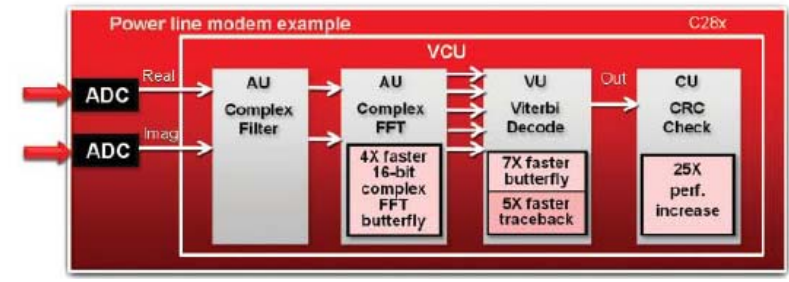

Figure 14. Performance speed-up on VCU [4]
The VCU II significantly accelerates functions like the IFFT and Reed-Solomon encoder in the TX signal path, and the FFT, channel equalizer, Viterbi decoder, Reed-Solomon decoder, and CRC in the RX signal path. The instruction set supports Viterbi decoders corresponding to constraint lengths (K) from 4 to 7 and code rates $R=1 / 2$ and $1 / 3$. The instruction set also supports CRC computation of different byte sizes like CRC8, CRC16, CRC24 and CRC32, which are used in PLC and IEEE 802.15.4g. The VCU II can also be used to improve the efficiency of algorithms such as complex correlation (used in SFD frame detection), narrow band interference detection, and channel estimation.

The following figure shows the improvement in speed of the standard MIPS intensive functions with VCU II over the standard C28x architecture. The comparative numbers of VCU I over the C28x architecture are also included for reference.

\section{IEEE 802.15.4g Modem Example}

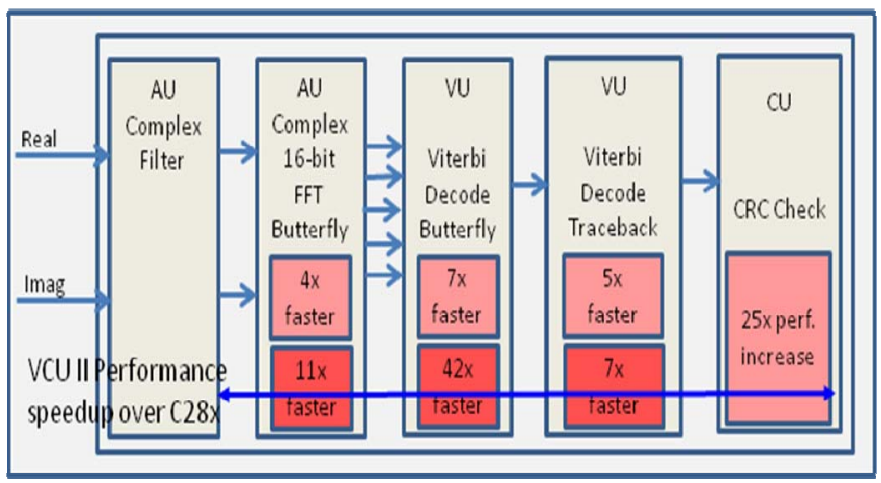

Figure 15. Performance speed-up on VCU II over C28x

\subsubsection{Control Law Accelerator (CLA)}

The floating-point CLA can be used for front-end channel filtering, including up sampling anti-aliasing filter on the TX chain and decimation filtering on the RX. It can also be used for implementing automatic gain control (AGC) and residual DC offset correction algorithms. Even though the CLA takes more cycles than the main C28x core, the CLA core provides additional MIPS and can be used to offload functions that directly interface with the CC1260.

\subsubsection{Trigonometric Math Unit (TMU)}

The TMU extends the capabilities of a C28x+ FPU to speed up the execution of common trigonometric and arithmetic operations like sqrt, sin, cos and atan [6].

\section{COMPLEXITY ANALYSIS}

The MIPS intensive communications algorithms, such as the FFT, used in OFDM, and the Viterbi decoder, used by all PHYs, can be implemented on the VCU II. Additional MIPS intensive communications algorithms for the OFDM PHY can be found in [7], while the MIPS intensive communications algorithms for the DSSS O-QPSK can be found in [8]. In this section, the complexity estimates of some of the communications algorithms running on the VCU II will be discussed. 


\subsection{Complex $2^{\mathrm{k}}$ FFT}

The IEEE 802.15.4g OFDM PHY uses a complex FFT that can be implemented efficiently using the tightly coupled instructions on the VCU II. A typical radix-2 butterfly computation is listed below. The additional instructions in the VCU II lead to a performance improvement that is close to $12 \times$ the cycle count on the C28x and nearly $3 \times$ improvement over the cycle count of the initial version of VCU. The VCU II instructions are based on implementing the radix-2 decimation-in-time by combining 4 radix-2 butterfly operations of 2 consecutive stages as shown in Figure 16.

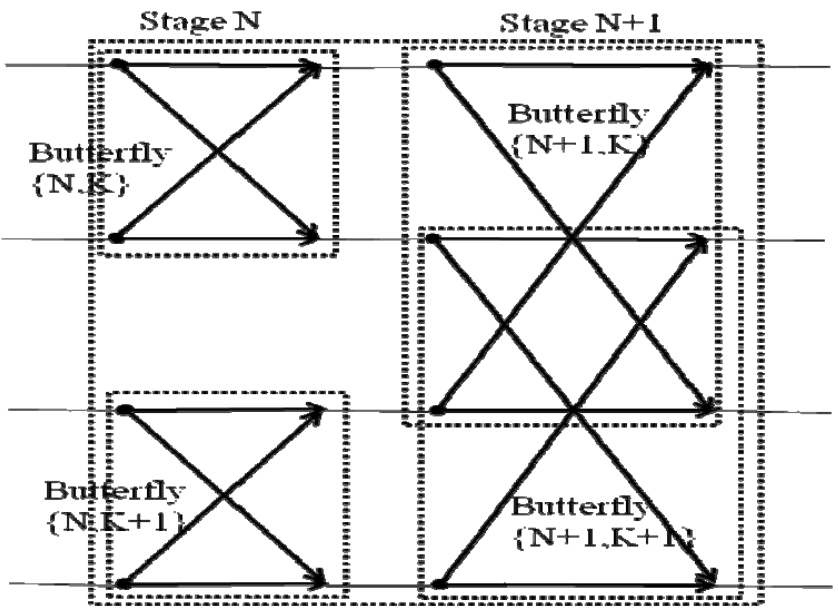

Figure 16. Combined 4 Radix-2 Butterflies of 2 adjacent stages

This results in a reduction in the number of read/write operations by a factor of 2 when compared to the conventional radix- 2 implementation. Additionally, only 2 twiddle factors need to be loaded compared to 4 for the intermediate stages and none for the first two stages. More generalized approaches that tradeoff computational speed, memory size. and access requirements can be found in [9]. The combined equations for the 4 butterflies of these first two stages are shown in Table 1.

Table 1. Combined equations for stages 1 \& 2 of Complex FFT

\begin{tabular}{|c|c|c|c|}
\hline & & Stages 1 \& 2 Imag & Stages 1 \& 2 Real \\
\hline I0 & R0 & $(\mathrm{I} 0+\mathrm{I} 1)+(\mathrm{I} 2+\mathrm{I} 3)$ & $(\mathrm{R} 0+\mathrm{R} 1)+(\mathrm{R} 2+\mathrm{R} 3)$ \\
$\mathrm{I} 1$ & $\mathrm{R} 1$ & $(\mathrm{I} 0-\mathrm{I} 1)-(\mathrm{R} 2-\mathrm{R} 3)$ & $(\mathrm{R} 0-\mathrm{R} 1)+(\mathrm{I} 2-\mathrm{I} 3)$ \\
I2 & R2 & $(\mathrm{I} 0+\mathrm{I} 1)-(\mathrm{I} 2+\mathrm{I} 3)$ & $(\mathrm{R} 0+\mathrm{R} 1)-(\mathrm{R} 2+\mathrm{R} 3)$ \\
I3 & R3 & $(\mathrm{I} 0-\mathrm{I} 1)+(\mathrm{R} 2-\mathrm{R} 3)$ & $(\mathrm{R} 0-\mathrm{R} 1)-(\mathrm{I} 2-\mathrm{I} 3)$ \\
\hline
\end{tabular}

The first two stages take 8 cycles for 4 butterflies and the remaining stages take 10 cycles for 4 butterflies. These instructions also facilitate a reduction in the total number of $\mathrm{read} / \mathrm{write}$ operations for both the data and the twiddle factor. The average number of cycles for each butterfly is approximately 2.5 cycles.

\subsection{Viterbi Decoder}

The MR-OFDM and MR-O-QPSK PHYs both use a K=7, R = $1 / 2$, convolutional code. The VCU II has sufficient internal memory to ensure that all the state metrics of the Viterbi decoder up to constraint length $\mathrm{K}=7$ can be stored internally and that all of the 32 butterfly operations for a given stage can be completed in parallel in a single cycle. Saving the state metrics internally is a huge improvement over VCU I where the state metrics had to be stored back into memory after each add-compare-select (ACS) butterfly operation.

The traceback operation requires 2 cycles for every output bit which is an improvement over the 3 cycles in the original VCU. This is accomplished by completing the traceback operation using the special VTRACE instruction in parallel with loading half of the information for the next output bit.

The inner loop for the butterfly computations of the ACS block for each stage can be completed in 3 cycles. This results in less than 3 MIPS for the highest data rate (800 kbps) in the IEEE 802.15.4g standard. The assembly code for the 3-cycle inner loop with the VCU-II instructions [10] is shown below:

$\begin{array}{ll}\text { RPTB__VITERBI_metricUpdateLoop, AR6 } \\ \text { VITSTAGE } & \text {; compute } 32 \text { butterflies in parallel } \\ \text { ॥VITBM2 VR0, *XAR0++ } & \text {; compute branch metric for next } \\ & \text {; stage } \\ \text { VMOV32 *XAR1++, VT1 } & \text {; store VT1 } \\ \text { VMOV32 *XAR1++, VT0 } & \text {; store VT0 } \\ \text { _ VITERBI_metricUpdateLoop: } & \end{array}$

The assembly code for the 2-cycle inner loop of the traceback operation is shown below. This loop is unrolled to generate 2 bits because of minimum code size restrictions of the RPTB instruction of C28x.

$\begin{array}{lll}\text { RPTB } & \text {-VITERBI_tracebackLoop, AR6 } \\ \text { VMOV32 } & \text { VT1, *--XAR3 } & \\ \text { VTRACE } & \text { VR1, VR0, VT0, VT1 } & \text {; traceback for bit 2k } \\ \text { |l VMOV32 VT0, *--XAR3 } & \text {; Load data for next bit } \\ \text { VMOV32 } & \text { VT1, *--XAR3 } & \\ \text { VTRACE } & \text { VR1, VR0, VT0, VT1 } & \text {; traceback for bit 2k+1 } \\ \text { II VMOV32 VT0, *-XAR3 } & \text {; Load data for next bit } \\ \text { - VITERBI_tracebackLoop: } & \\ \text { VREVB } & \text { VR1 }\end{array}$

\subsection{Cyclic Redundancy Check (CRC)}

The VCU II can compute the CRC, used by the IEEE 802.15.4g standard, for a block length of 10 bytes in 10 cycles [4]. Note that the CRC is denoted as Frame Check Sequence (FCS) in the standard.

\subsection{Frequency Offset Correction}

The VCU II has specific instructions to accelerate complex multiply operations. The frequency correction algorithm, that is common to all PHYs, uses a complex multiply to correct for phase/frequency rotation that takes about 12 cycles for each sample. The inner loop of the DSP assembly code is listed below. This example clearly shows the mixture between the baseline 
C28x instructions and the newer VCU-II specific instructions, including the register moves and memory read and write operations from the VCU II registers.

\begin{tabular}{lll} 
RPTB & _PHY_derot_loop1, AR1 & \\
VCCMPY & VR3, VR2, VR1, VR0 & \\
\multicolumn{1}{|l}{ VMOV32 } & VR1, *XAR7++ & ; VR1=x1 \\
ADD & AL, AR2 & ; AL+=offset \\
AND & AH, AL, \#0xffc0 & ; assume LUT size 128 \\
LSR & AH, 5 & ; AR0=table index \\
MOV & AR0, AH & \\
VMOV32 & VR0, *+XAR6[AR0] & \\
\multicolumn{1}{l}{$\|$ VCDADD16 VR5, VR4, VR3, VR2 } & \\
VMOV32 & *XAR3++, VR5 & \\
_PHY_derot_loop1: & ; store real \\
MOV & *+XAR4[1], AL &
\end{tabular}

\subsection{MIPS for FSK, OFDM and DSSS}

Based on earlier analysis, we summarize the MIPS requirement for the primary blocks in the different PHYs as a function of the channel spacing and data rate.

\subsubsection{MIPS for FFT in OFDM}

The FFT is used only in the OFDM PHY. The MIPS requirement for the FFT is dependent on the number of tones and the OFDM symbol length. Note that this number is completely independent of the specific data rate in any given option. As discussed earlier in Section 3.2, we need to compute 25/3k FFT/sec for all the four options.

Table 2 FFT MIPS usage for OFDM Options

\begin{tabular}{|c|c|c|c|c|}
\hline Parameter & Option 1 & Option 2 & Option 3 & Option 4 \\
\hline DFT Size & 128 & 64 & 32 & 16 \\
\hline FFT MIPS & 11 & 5 & 3 & 1.5 \\
\hline
\end{tabular}

\subsubsection{MIPS for Viterbi Decoder}

The MIPS requirement for the Viterbi Decoder and FCS computation are dependent only on the final data rate and are independent of the channel bandwidth. Table 3 - Table 5 summarize the MIPS requirement for the OFDM, FSK and DSSS PHYs, respectively.

Table 3 Viterbi Decoder MIPS usage for OFDM Rates

\begin{tabular}{|c|c|c|c|c|c|}
\hline $\begin{array}{c}\text { Data Rate } \\
\text { (kbps) }\end{array}$ & $\mathbf{5 0}$ & $\mathbf{1 0 0}$ & $\mathbf{2 0 0}$ & $\mathbf{4 0 0}$ & $\mathbf{8 0 0}$ \\
\hline $\begin{array}{c}\text { Viterbi Decoder } \\
\text { MIPS }\end{array}$ & 0.5 & 1 & 2 & 4 & 8 \\
\hline
\end{tabular}

Table 4 Viterbi Decoder MIPS usage for some FSK Rates

\begin{tabular}{|c|c|c|c|c|c|}
\hline Data Rate (kbps) & $\mathbf{2 . 4}$ & $\mathbf{1 0}$ & $\mathbf{5 0}$ & $\mathbf{2 0 0}$ & $\mathbf{4 0 0}$ \\
\hline $\begin{array}{c}\text { Viterbi Decoder } \\
\text { MIPS }\end{array}$ & $\sim 0$ & $<0.5$ & 0.5 & 2 & 4 \\
\hline
\end{tabular}

Table 5 Viterbi Decoder MIPS for DSSS (O-QPSK) Rates

\begin{tabular}{|c|c|c|c|c|c|}
\hline $\begin{array}{c}\text { Data Rate } \\
\text { (kbps) }\end{array}$ & $\mathbf{3 1 . 2 5}$ & $\mathbf{6 2 . 5}$ & $\mathbf{1 2 5}$ & $\mathbf{2 5 0}$ & $\mathbf{5 0 0}$ \\
\hline $\begin{array}{c}\text { Viterbi Decoder } \\
\text { MIPS }\end{array}$ & 0.5 & 1 & 1.5 & 2.5 & 5 \\
\hline
\end{tabular}

Without the VCU II, the MIPS requirement for OFDM Option 1 (128-point FFT, 1.2 MHz channel spacing) and a data rate of 800 kbps would be $>200$ MIPS for just the FFT and Viterbi decoder. This clearly shows the need for an ASIP type of instruction enhancement provided by the VCU II.

\subsubsection{MIPS for Frequency Offset Correction}

The MIPS requirement for the frequency offset correction algorithm is dependent on the sample rate and the channel spacing. Assuming a $2 \times$ oversampling rate, Table 6 summarizes the MIPS requirement for the different OFDM options.

Table 6 OFDM Frequency Offset Correction MIPS

\begin{tabular}{|c|c|c|c|c|}
\hline Parameter & Option 1 & Option 2 & Option 3 & Option 4 \\
\hline $\begin{array}{c}\text { Sample Rate } \\
\text { (Msps) }\end{array}$ & $4 / 3$ & $2 / 3$ & $1 / 3$ & $1 / 6$ \\
\hline $\begin{array}{c}\text { FO } \\
\text { Correction } \\
\text { MIPS }\end{array}$ & 11 & 6 & 3 & 1.5 \\
\hline
\end{tabular}

Table 7 shows the MIPS requirements for the frequency offset correction for an FSK PHY assuming a $2 \times$ oversampling factor.

Table 7 FSK Frequency Offset Correction MIPS

\begin{tabular}{|c|c|c|c|c|c|}
\hline $\begin{array}{c}\text { Channel Spacing } \\
\text { (kHz) }\end{array}$ & $\mathbf{1 2 . 5}$ & $\mathbf{2 5}$ & $\mathbf{2 0 0}$ & $\mathbf{4 0 0}$ & $\mathbf{6 0 0}$ \\
\hline Sample Rate (Msps) & 0.025 & 0.05 & 0.4 & 0.8 & 1.2 \\
\hline FO Correction MIPS & 0.5 & 0.5 & 4 & 7 & 10 \\
\hline
\end{tabular}

Table 8 lists the MIPS requirements for the frequency offset correction for O-QPSK assuming a $2 \times$ oversampling factor.

Table 8 O-QPSK Frequency Offset Correction MIPS

\begin{tabular}{|c|c|c|c|}
\hline Parameter & 100 kcps & 1000 kcps & 2000 kcps \\
\hline Sample Rate (Msps) & $1 / 5$ & 2 & 4 \\
\hline FO Correction MIPS & 2 & 16 & 32 \\
\hline
\end{tabular}




\section{SUMMARY}

In this paper, we have provided an overview of a software-defined radio platform for smart utility networks based on the IEEE 802.15.4g standard. This platform can implement any of the three PHYs as well as several proprietary legacy radios that already exist in the market place. The SDR provides a flexible platform that can implement existing technology, while offering an easy upgrade path as the technology evolves. This flexible platform can also be used to meet the various throughput requirements, cost and power requirements, and frequency bands and channelizations available around the world. The DSP on the SDR platform is also flexible enough to allow additional standardsbased and proprietary PHY implementations.

\section{REFERENCES}

[1] IEEE 802.15.4g Physical Layer (PHY) Specifications for Low-Data-Rate, Wireless, Smart Metering Utility Networks, April 2012.

[2] Flammer, G., Monnerie, E., Shearer, S. and Shimada, S. 2009 April. "Channel Characterization for SUN", Submission to IEEE P802.15 TG4g CFP https://mentor.ieee.org/802.15/dcn/08/15-09-0279-01-004gchannel-characterization-for-sun.ppt

[3] Palkovic, M., Raghavan, P., Li, Min, Dejonghe, A., Van der Perre, L. and Catthoor, F. 2010 March. "Future SoftwareDefined Radio Platforms and Mapping Flows”. IEEE Signal Processing Magazine. pp 22-33.
[4] Tessarolo, A. and Chowdhury, P. 2011 January. "Bringing efficient communications to Real-time motor control and power conversion applications with TI's Viterbi Complex Math Unit (VCU)”. Texas Instruments White Paper. http://www.ti.com/lit/wp/spry158/spry158.pdf

[5] Xu, G., Yim, S., Kim, I. H., Pande, T and Lu, X. 2012. "Implementation and Field Test Results of a Software Defined PLC Modem”. In IEEE International Symposium on Power Line Communications and Its Applications.

[6] Texas Instruments, TMS320F2837xD Dual-Core Delfino ${ }^{\mathrm{TM}}$ Microcontrollers http://www.ti.com/lit/ds/sprs880a/sprs880a.pdf

[7] Bloessl, B., Segata, M., Sommer, C. and Dressler, F. 2013 August. "An IEEE 802.11a/g/p OFDM Receiver for GNU Radio”. In ACM SIGCOMM Software Radio Implementation Forum.

[8] M. Schmidt, "A first look at the performance of SUN DSSS,” IEEE P802.15-09-0744-00-004g, November 2009

[9] Baas, B. 2005. "A generalized cached-FFT algorithm”. In IEEE Acoustics, Speech and Signal Processing, ICASSP 2005 pp. V-89-V-92

[10] Texas Instruments, “TMS320C28x Extended Instruction Sets Technical Reference Manual”, March 2014 http://www.ti.com/lit/ug/spruhs1/spruhs1.pdf 